\title{
Elevada prevalência de fatores de risco para doenças crônicas entre universitários
}

\author{
High prevalence of risk factors for chronic diseases among university students
}

\author{
Barbara Virginia Caixeta Crepaldia, Hayanny Pires Netto Guimarães ${ }^{b}$, Cinthia Domingos Barbosa ${ }^{a}$, \\ Larissa Santos Molinac, Lycea Maria Maciel Nogueirac, Luana Padua Soares ${ }^{\mathrm{d}}$ \\ a Nutricionista. Mestranda do Programa de Pós-Graduação em Ciências da Saúde, Faculdade de Medicina, Universidade Federal de Uberlândia, Minas Gerais, Brasil. \\ b Nutricionista graduada pela Universidade Federal de Uberlândia. Residente do Programa de Residência Integrada Multiprofissional em Saúde - Saúde do Idoso, \\ Universidade Federal do Triângulo Mineiro, Minas Gerais, Brasil. \\ c Nutricionista graduada pela Universidade Federal de Uberlândia, Minas Gerais, Brasil. \\ d Nutricionista. Mestre em Saúde Pública. Doutora em Saúde na Comunidade. Docente do Curso de Graduação em Nutrição, Faculdade de Medicina, \\ Universidade Federal de Uberlândia, Minas Gerais, Brasil.
}

RESUMO Objetivo: Este estudo avaliou a prevalência de fatores de risco compartilhados e modificáveis para doenças crônicas não transmissíveis entre estudantes de uma universidade pública brasileira.

Materiais e Métodos: Participaram do estudo 379 alunos de diferentes áreas de conhecimento e períodos. Os dados foram coletados por meio de questionário autoaplicável, contendo questões sobre o âmbito acadêmico, dados socioeconômicos, estado nutricional e estilo de vida.

Resultados: No total, 95,4\% dos estudantes apresentaram pelo menos três fatores de risco, sendo os mais comuns o baixo consumo de frutas, hortaliças e leite, o consumo de carnes com excesso de gordura, a ingestão de bebidas alcoólicas, a ausência/carência de exercícios físicos e o gasto excessivo de tempo com eletrônicos. Observaram-se diferenças significativas de comportamento em relação a gênero, área de conhecimento e período.

Conclusão: Os resultados evidenciam um perfil preocupante entre os universitários e demonstram a necessidade de implementação de programas educativos no âmbito acadêmico, a fim de reduzir a exposição dos alunos aos fatores de risco para doenças crônicas não transmissíveis.

Palavras-chave: doenças crônicas; estilo de vida; estudantes.

Objective: This study evaluated the prevalence of shared and modifiable risk factors for chronic noncommunicable diseases among students in a Brazilian public university.

Materials and Methods: The study included 379 students from different areas of knowledge and periods. Data were collected through self-administered questionnaire with questions about the academic, socioeconomic data, nutritional status and lifestyle.

Results: In total, 95.4\% of students had at least three risk factors, the most common being the low consumption of fruits, vegetables and milk, meat consumption with excess fat, alcohol consumption, lack / shortage physical exercise and excessive spending time with electronics. There were significant differences in behavior in relation to gender, area of expertise and periods.

Conclusion: The results show a worrying profile among university and demonstrate the need to implement educational programs in the University, in order to reduce the exposure of students to the risk factors for chronic noncommunicable diseases.

Keywords: chronic diseases; lifestyles; students. 


\section{INTRODUÇÃO}

As Doenças Crônicas Não Transmissíveis (DCNT) são as principais causas de morte no mundo, totalizando $63 \%$ dos óbitos em 2008 ${ }^{1}$. No Brasil, elas constituem o problema de saúde de maior magnitude e correspondem a $72 \%$ das causas de morte ${ }^{2}$. Dentre as DCNT destacam-se as doenças do aparelho circulatório principalmente a hipertensão arterial sistêmica (HAS), além do câncer, diabetes mellitus (DM) e doenças respiratórias crônicas ${ }^{1}$.

Essas doenças possuem fatores de risco modificáveis em comum, tais como, o tabagismo, consumo nocivo de bebida alcoólica, sedentarismo, alimentação inadequada e obesidade ${ }^{3}$. As doenças e agravos não transmissíveis têm expressão clínica após longo tempo de exposição aos fatores de risco e da convivência assintomática do indivíduo ${ }^{4}$. A população jovem, destacando-se a universitária, está exposta a vários destes fatores de risco.

Os universitários estão sujeitos a uma série de alterações fisiológicas, típicas da juventude, além das possíveis mudanças sociológicas e culturais, devido ao início da faculdade, deixando a casa da família em diversas ocasiões, intensa sensação de liberdade e autonomia, início de uma construção de futuro seguindo as perspectivas idealizadas, entre outros ${ }^{5,6}$.

Nesse contexto, o meio universitário proporciona novas relações sociais, bem como o reforço de novos comportamentos e hábitos, tornando os indivíduos vulneráveis às condutas de risco a saúde. Todas essas mudanças influenciam de forma direta o estilo de vida dos universitários, que poderá ser levado por toda sua vida ou neles permanecer por tempo suficiente para afetar sua saúde. Atualmente, é possível que o estilo de vida seja um dos mais importantes determinantes de saúde da população estudantil ${ }^{5,6,7,8}$.

Estudos demonstram que, de um modo geral, universitários não apresentam um estilo de vida saudável ${ }^{7,8} \mathrm{e}$ que a maioria deles manifesta três ou mais comportamentos de risco para DM, HAS e doenças cardiovasculares ${ }^{9}$. É importante salientar que os fatores de risco para DCNT são propensos a se somarem ${ }^{10}$.

Diante do exposto, é fundamental a avaliação de comportamentos de risco para DCNT entre universitários, a fim de permitir a prevenção e até mesmo intervenção em curto e longo prazo. A universidade é um ambiente propício para a criação de estratégias de promoção da saúde, prevenção e controle de doenças, influenciando os estudantes a almejar um estilo de vida saudável. O objetivo do trabalho foi avaliar a prevalência de fatores de risco compartilhados e modificáveis para DCNT entre estudantes de uma universidade pública de Uberlândia, Minas Gerais.

\section{MATERIAIS E MÉTODOS}

Trata-se de um estudo observacional transversal, desenvolvido no período de agosto de 2013 a abril de 2014, com alunos de uma universidade pública do município de Uberlândia, Minas Gerais, Brasil.

O cálculo amostral foi realizado a partir do número de alunos matriculados na Universidade Federal de Uberlândia, em cursos de graduação oferecidos nos campi de Uberlândia (aproximadamente 17.866). Considerou-se um erro amostral de $5 \%(E=0,05)$, sendo obtida uma amostra de 392 alunos. As perdas referiram-se a estudantes que não estavam presentes nos dias de coleta de dados, universitários que não quiseram participar e questionários incompletos que foram excluídos da tabulação. Ao final, obteve-se uma amostra composta de 379 alunos, que corresponde a 96,7\% da amostra inicialmente definida.

Na seleção da amostra, foram considerados alunos das diferentes áreas do conhecimento: Ciências Biológicas, Agrárias e da Saúde; Engenharias, Ciências Exatas e da Terra; Ciências Humanas, Sociais, Aplicadas, Linguística, Letras e Artes. Em cada uma das grandes áreas, foram sorteados dois cursos. Em cada um dos cursos, foram selecionados alunos matriculados no início e no final dos mesmos. Como início, foram considerados o $1^{\mathrm{O}}$ e $2^{\mathrm{o}}$ períodos (para cursos semestrais) ou o primeiro ano (para cursos anuais). Como final, foram considerados o penúltimo e último períodos (para cursos semestrais) ou o último ano (para cursos anuais), sendo desconsiderados períodos de estágio e internato. Todos os alunos dos cursos e períodos selecionados foram convidados a participar da pesquisa. Foram incluídos no estudo os universitários selecionados para a pesquisa, regularmente matriculados na universidade, com idade igual ou superior a 18 anos e que assinaram o Termo de Consentimento Livre e Esclarecido (TCLE). Foram excluídas as alunas gestantes.

$\mathrm{O}$ instrumento de coleta de dados fundamentou-se em um questionário construído com base nos instrumentos utilizados em outras pesquisas, sendo autoaplicável. Foram incluídos itens referentes ao âmbito acadêmico (como curso e período), questões sobre dados socioeconômicos dos estudantes, estado nutricional (peso e altura referidos) e estilo de vida.

Para avaliar o estado nutricional calculou-se o Índice de Massa Corporal (IMC) por meio da fórmula: [peso referido $(\mathrm{kg}) /$ altura referida $\left.(\mathrm{m})^{2}\right]^{11}$. A fim de avaliar o padrão de consumo alimentar, foi realizada uma adaptação do questionário do Sistema de Vigilância Alimentar e Nutricional (SISVAN) ${ }^{12}$. As questões referentes à prática de exercícios físicos e comportamentos sedentários, hábito de fumar e consumo de bebidas alcoólicas foram adaptadas do questionário de Vigilância de Fatores de Risco e Proteção 
para Doenças Crônicas por Inquérito Telefônico (VIGITEL) ${ }^{13}$ e questionário da Pesquisa Nacional de Saúde do Escolar (PENSE) ${ }^{14}$. As definições dos fatores de risco para DCNT considerados no estudo encontram-se na Tabela 1. Uma vez que os comportamentos de risco para DCNT tendem a se somarem, analisou-se também a soma dos fatores de risco entre os universitários.

Realizou-se análise descritiva dos dados para verificar frequências, bem como médias e desvio padrão. Utilizou-se o teste de Kolmogorov-Smirnov para verificar a normalidade das variáveis. O teste Qui-quadrado e o teste exato de Fisher foram utilizados para comparar prevalências e o teste t de Student e análise de variância (ANOVA) para comparar médias. Os dados foram analisados por meio dos softwares Statistical Package for Social Sciences (SPSS) versão 17 e Epi Info versão 3.5.3, sendo fixado um nível de significância de $5 \%$ quando testadas hipóteses.

O estudo foi aprovado pelo Comitê de Ética em Pesquisa da Universidade Federal de Uberlândia (Número do Parecer: 538.984).

Tabela 1. Definição dos fatores de risco para DCNT.

\begin{tabular}{|c|c|c|}
\hline Tópico & Fator de Risco & Indicadores \\
\hline Estado Nutricional* & Excesso de peso & $\%$ de universitários que apresentaram IMC $\geq 25,0 \mathrm{~kg} / \mathrm{m}^{2}$ \\
\hline Bebida Alcoólica & Consumo de bebidas alcoólicas & $\begin{array}{l}\text { \% de universitários que referiram beber, independentemente do tipo de bebida } \\
\text { alcoólica, da frequência e quantidade de ingestão. }\end{array}$ \\
\hline Tabagismo & Hábito de fumar & $\begin{array}{l}\text { \% de universitários que referiram fumar, independentemente do tipo de cigarro, } \\
\text { da frequência e intensidade do hábito de fumar. }\end{array}$ \\
\hline \multirow[t]{2}{*}{ Comportamento Sedentário** } & $\begin{array}{l}\text { Ausência/carência de exercícios } \\
\text { físicos }\end{array}$ & $\begin{array}{l}\text { \% dos universitários que referiram não praticar algum tipo de exercício físico } \\
\text { e aqueles que afirmaram realizar, porém por um tempo inferior a } 30 \text { minutos } \\
\text { e com uma frequência semanal menor que três vezes. }\end{array}$ \\
\hline & $\begin{array}{l}\text { Gasto excessivo de tempo } \\
\text { com eletrônicos }\end{array}$ & $\begin{array}{l}\text { \% de universitários que referiram despender em um dia de semana comum, } \\
\text { três ou mais horas diárias vendo televisão e/ou utilizando computador e/ou } \\
\text { jogando videogame. }\end{array}$ \\
\hline \multirow[t]{10}{*}{ Consumo Alimentar*** } & $\begin{array}{l}\text { Ingestão de carnes com excesso } \\
\text { de gordura }\end{array}$ & $\begin{array}{l}\text { \% de universitários que referiram ter o hábito de ingestão de carne vermelha } \\
\text { e frango com excesso de gordura, sem retirada da gordura visível e a pele, } \\
\text { respectivamente. }\end{array}$ \\
\hline & $\begin{array}{l}\text { Consumo excessivo de bebidas } \\
\text { açucaradas }\end{array}$ & $\begin{array}{l}\text { \% de universitários que referiram ingerir refrigerantes e/ou sucos artificiais pelo } \\
\text { menos cinco dias por semana. }\end{array}$ \\
\hline & $\begin{array}{l}\text { Ingestão excessiva de alimentos } \\
\text { embutidos }\end{array}$ & $\begin{array}{l}\text { \% de universitários que referiram ingerir alimentos embutidos pelo menos } \\
\text { cinco dias por semana. }\end{array}$ \\
\hline & $\begin{array}{l}\text { Ingestão excessiva de alimentos } \\
\text { fritos }\end{array}$ & $\begin{array}{l}\% \text { de universitários que referiram ingerir alimentos fritos pelo menos cinco dias } \\
\text { por semana. }\end{array}$ \\
\hline & $\begin{array}{l}\text { Ingestão excessiva de alimentos } \\
\text { doces }\end{array}$ & $\begin{array}{l}\text { \% de universitários que referiram ingerir alimentos doces pelo menos cinco dias } \\
\text { por semana. }\end{array}$ \\
\hline & Baixo consumo de feijão & \% de universitários que referiram consumir feijão inferior a cinco dias por semana. \\
\hline & Baixo consumo de frutas & $\begin{array}{l}\text { \% de universitários que referiram consumir frutas/salada de frutas ou sucos naturais } \\
\text { de frutas menos que sete dias por semana. }\end{array}$ \\
\hline & Baixo consumo de hortaliças & $\begin{array}{l}\text { \% de universitários que referiram consumir hortaliças (exceto batata, mandioca, } \\
\text { inhame e cará) menos que sete dias por semana. }\end{array}$ \\
\hline & Baixo consumo de leite & \% de universitários que referiram consumir leite menos que sete dias por semana. \\
\hline & $\begin{array}{l}\text { Adição de sal na comida depois } \\
\text { de pronta }\end{array}$ & $\begin{array}{l}\text { \% de universitários que referiram ter o hábito de adicionar sal na comida depois } \\
\text { de pronta. }\end{array}$ \\
\hline
\end{tabular}

* Avaliado segundo os critérios estabelecidos pela World Health Organization (1990) ${ }^{11}$. ** Prática de exercícios físicos avaliado de forma semelhante aos critérios do American College of Sports Medicine ${ }^{15}$, e tempo gasto em eletrônicos avaliado segundo critérios adotados pelo VIGITEL $2013^{13}$. ${ }^{* * *}$ Avaliado de acordo com os critérios estabelecidos pelo Ministério da Saúde (10 Passos para uma Alimentação Saudável) ${ }^{16}$, VIGITEL $2013^{13}$ e PENSE $2012^{14}$. 


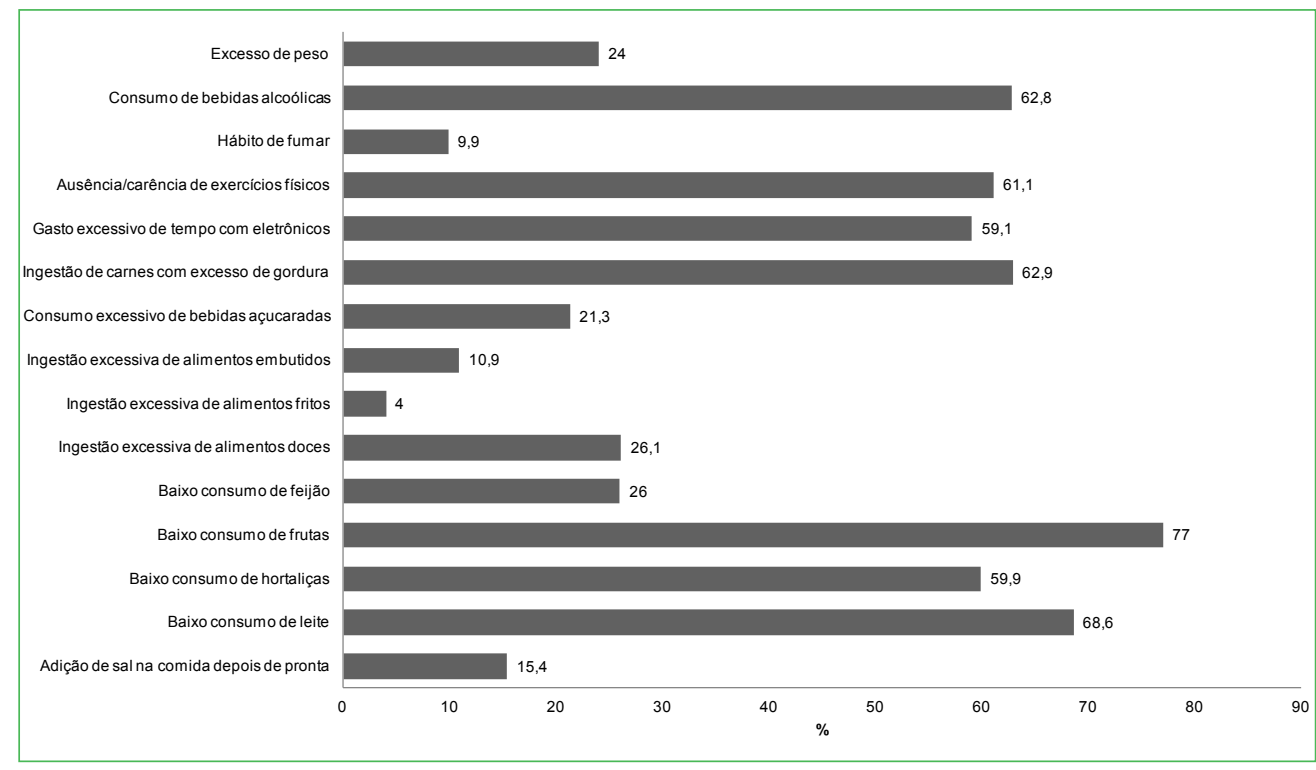

Figura 1. Distribuição dos universitários segundo os fatores de risco para DCNT. foram o baixo consumo de frutas, hortaliças e leite, consumo de carnes com excesso de gordura, ingestão de bebidas alcoólicas, ausência/carência de exercícios físicos e gasto excessivo de tempo com eletrônicos.

Ao comparar os gêneros, de um modo geral, os homens apresentaram significativamente mais fatores de risco para DCNT, destacando-se o excesso de peso, hábito de fumar, ingestão de carnes com excesso de gordura, consumo excessivo de bebidas açucaradas e baixo consumo de frutas. Diferentemente, quanto ao consumo excessivo de doces e a baixa ingestão de feijão, observou-se uma prevalência maior entre as mulheres (valor de $p<0,05$ ), como apresentado na Tabela 2.

A Tabela 3 evidencia diferenças significativas entre as áreas de conhecimento em relação ao hábito de fumar, gasto excessivo de tempo com eletrônicos, consumo excessivo de bebidas açucaradas, baixo consumo de feijão e leite. Destaca-se que dos cinco fatores de risco mencionados, três tiveram maior prevalência na $\mathrm{A} 2$, seguida da $\mathrm{A} 3$ e por fim da A1. O hábito de fumar e o baixo consumo de feijão por parte dos universitários apresentaram diferente distribuição entre as áreas de conhecimento $(\mathrm{A} 3>\mathrm{A} 1>\mathrm{A} 2)$.

Tabela 2. Distribuição absoluta e percentual dos universitários, segundo fatores de risco para DCNT, por sexo.

\begin{tabular}{|c|c|c|c|c|c|}
\hline \multirow{3}{*}{ Fatores de Risco } & \multicolumn{4}{|c|}{ Sexo } & \multirow{3}{*}{ Valor de $p$} \\
\hline & \multicolumn{2}{|c|}{ Feminino } & \multicolumn{2}{|c|}{ Masculino } & \\
\hline & n & $\%$ & n & $\%$ & \\
\hline Excesso de peso & 20 & 10,4 & 70 & 38,5 & $0,000^{\#}$ \\
\hline Consumo de bebidas alcoólicas & 118 & 60,2 & 120 & 65,6 & 0,290 \\
\hline Hábito de fumar & 4 & 2,1 & 33 & 18,3 & $0,000^{\#}$ \\
\hline Ausência/carência de exercícios físicos & 123 & 63,1 & 108 & 59,0 & 0,460 \\
\hline Gasto excessivo de tempo com eletrônicos & 116 & 60,1 & 104 & 58,1 & 0,752 \\
\hline Ingestão de carnes com excesso de gordura & 106 & 54,4 & 131 & 72,0 & $0,000^{\#}$ \\
\hline Consumo excessivo de bebidas açucaradas & 30 & 15,3 & 50 & 27,8 & $0,004^{\#}$ \\
\hline Ingestão excessiva de alimentos embutidos & 16 & 8,2 & 25 & 13,7 & 0,099 \\
\hline Ingestão excessiva de alimentos fritos & 5 & 2,6 & 10 & 5,5 & 0,189 \\
\hline Ingestão excessiva de alimentos doces & 71 & 36,2 & 28 & 15,3 & $0,000^{\#}$ \\
\hline Baixo consumo de feijão & 62 & 32,3 & 33 & 19,0 & $0,004^{\#}$ \\
\hline Baixo consumo de frutas & 140 & 71,4 & 151 & 83,0 & $0,010^{\#}$ \\
\hline Baixo consumo de hortaliças & 109 & 55,6 & 118 & 64,5 & 0,093 \\
\hline Baixo consumo de leite & 135 & 68,9 & 125 & 68,3 & 0,912 \\
\hline Adição de sal na comida depois de pronta & 28 & 14,3 & 30 & 16,6 & 0,570 \\
\hline
\end{tabular}

* Teste Exato de Fisher. ${ }^{*}$ Valor de $\mathrm{p}<0,05$. 
A prevalência dos fatores de risco em universitários de acordo com o período em que se encontravam matriculados pode ser observada na Tabela 4. Os estudantes apresentaram maiores percentuais de ausência/ carência de exercícios físicos, consumo excessivo de bebidas açucaradas e ingestão excessiva de alimentos doces no início da graduação. Também houve diferença significativa para o excesso de peso, apresentando-se maior entre os universitários que se encontravam no final da graduação.

Tabela 3. Distribuição absoluta e percentual dos universitários, segundo fatores de risco para DCNT, por área de conhecimento.

\begin{tabular}{|c|c|c|c|c|c|c|c|}
\hline \multirow{3}{*}{ Fatores de Risco } & \multicolumn{6}{|c|}{ Área de Conhecimento } & \multirow{3}{*}{ Valor de $\mathrm{p}^{*}$} \\
\hline & \multicolumn{2}{|c|}{ A1 } & \multicolumn{2}{|c|}{ A2 } & \multicolumn{2}{|c|}{ A3 } & \\
\hline & $\mathbf{n}$ & $\%$ & $\mathrm{n}$ & $\%$ & $\mathbf{n}$ & $\%$ & \\
\hline Excesso de peso & 49 & 24,4 & 14 & 25,5 & 27 & 22,7 & 0,909 \\
\hline Consumo de bebidas alcoólicas & 129 & 63,5 & 33 & 60,0 & 76 & 62,8 & 0,890 \\
\hline Hábito de fumar & 16 & 7,9 & 1 & 1,9 & 20 & 16,5 & $0,005^{\#}$ \\
\hline Ausência/carência de exercícios físicos & 128 & 63,1 & 33 & 60,0 & 70 & 58,3 & 0,690 \\
\hline Gasto excessivo de tempo com eletrônicos & 91 & 45,5 & 44 & 80,0 & 85 & 72,6 & $0,000^{\#}$ \\
\hline Ingestão de carnes com excesso de gordura & 134 & 66,3 & 32 & 59,3 & 71 & 58,7 & 0,324 \\
\hline Consumo excessivo de bebidas açucaradas & 32 & 16,0 & 19 & 34,5 & 29 & 24,0 & $0,008^{\#}$ \\
\hline Ingestão excessiva de alimentos embutidos & 26 & 12,8 & 4 & 7,4 & 11 & 9,2 & 0,404 \\
\hline Ingestão excessiva de alimentos fritos & 5 & 2,5 & 4 & 7,4 & 6 & 5,0 & 0,202 \\
\hline Ingestão excessiva de alimentos doces & 48 & 23,6 & 11 & 20,0 & 40 & 33,1 & 0,094 \\
\hline Baixo consumo de feijão & 48 & 24,7 & 7 & 13,5 & 40 & 33,3 & $0,021^{\#}$ \\
\hline Baixo consumo de frutas & 154 & 75,9 & 44 & 81,5 & 93 & 76,9 & 0,683 \\
\hline Baixo consumo de hortaliças & 119 & 58,6 & 27 & 49,1 & 81 & 66,9 & 0,070 \\
\hline Baixo consumo de leite & 126 & 62,1 & 45 & 81,8 & 89 & 73,6 & $0,007^{\#}$ \\
\hline Adição de sal na comida depois de pronta & 28 & 13,8 & 7 & 12,7 & 23 & 19,3 & 0,347 \\
\hline
\end{tabular}

A1 (Ciências Biológicas, Agrárias e da Saúde); A2 (Engenharias, Ciências Exatas e da Terra); A3 (Ciências Humanas, Sociais, Aplicadas, Linguística, Letras e Artes).

* Teste qui-quadrado. ${ }^{*}$ Valor de $\mathrm{p}<0,05$.

Tabela 4. Distribuição absoluta e percentual dos universitários, segundo fatores de risco para DCNT, por período.

\begin{tabular}{|c|c|c|c|c|c|}
\hline \multirow{3}{*}{ Fatores de Risco } & \multicolumn{4}{|c|}{ Período } & \multirow{3}{*}{ Valor de $\mathrm{p}^{*}$} \\
\hline & \multicolumn{2}{|c|}{ Início' ${ }^{1}$} & \multicolumn{2}{|c|}{ Fim $^{2}$} & \\
\hline & $\mathbf{n}$ & $\%$ & $\mathbf{n}$ & $\%$ & \\
\hline Excesso de peso & 36 & 18,6 & 54 & 29,8 & $0,011^{\#}$ \\
\hline Consumo de bebidas alcoólicas & 117 & 59,4 & 121 & 66,5 & 0,167 \\
\hline Hábito de fumar & 25 & 12,7 & 12 & 6,7 & 0,058 \\
\hline Ausência/carência de exercícios físicos & 133 & 67,9 & 98 & 53,8 & $0,006^{\#}$ \\
\hline Gasto excessivo de tempo com eletrônicos & 114 & 59,4 & 106 & 58,9 & 1,000 \\
\hline Ingestão de carnes com excesso de gordura & 120 & 60,9 & 117 & 65,0 & 0,455 \\
\hline Consumo excessivo de bebidas açucaradas & 53 & 26,9 & 27 & 15,1 & $0,006^{\#}$ \\
\hline Ingestão excessiva de alimentos embutidos & 20 & 10,3 & 21 & 11,5 & 0,742 \\
\hline Ingestão excessiva de alimentos fritos & 8 & 4,1 & 7 & 3,8 & 1,000 \\
\hline Ingestão excessiva de alimentos doces & 65 & 33,0 & 34 & 18,7 & $0,002^{\#}$ \\
\hline Baixo consumo de feijão & 49 & 25,7 & 46 & 26,3 & 0,906 \\
\hline Baixo consumo de frutas & 157 & 80,1 & 134 & 73,6 & 0,144 \\
\hline Baixo consumo de hortaliças & 122 & 61,9 & 105 & 57,7 & 0,404 \\
\hline Baixo consumo de leite & 132 & 67,0 & 128 & 70,3 & 0,508 \\
\hline Adição de sal na comida depois de pronta & 33 & 16,8 & 25 & 13,8 & 0,476 \\
\hline
\end{tabular}


Considerando a somatória de fatores de risco, os universitários apresentaram de 0 a $14(5,9 \pm 2,1)$. No total, 95,4\% dos estudantes tinham pelo menos três fatores de risco. Quanto aos gêneros, os homens apresentaram média de comportamento de risco $(6,2 \pm 2,1)$ significativamente maior que as mulheres $(5,6 \pm 2,0)(p=0,003)$. Os fatores de risco tiveram maior média nos alunos da $\mathrm{A} 3(6,3 \pm 2,2)$, seguida da A2 $(5,9 \pm 1,9)$ e por fim da A1 $(5,6 \pm 2,1)$ $(p=0,017)$. Em relação ao período matriculado, não houve diferença significativa entre a média dos fatores de risco no início $(6,1 \pm 2,2)$ e final do curso $(5,7 \pm 2,0)$.

\section{DISCUSSÃO}

Os resultados evidenciam um perfil preocupante entre os universitários, compatível com risco para algumas DCNT. Em sete comportamentos de risco avaliados percebe-se a presença destes em mais da metade dos alunos.

Em relação à alimentação, destacou-se o baixo consumo de frutas, cuja frequência encontrada (77\%) corrobora com outra pesquisa realizada com universitários, que constatou prevalência de $81,2 \%{ }^{17}$. Além disso, este mesmo estudo identificou o baixo consumo de hortaliças em $57 \%$ dos alunos avaliados ${ }^{17}$, indo ao encontro do observado na presente pesquisa (59,9\%).

A prevalência acentuada do baixo consumo de leite $(68,6 \%)$ é preocupante, pois a ingestão desta bebida pode ser inversamente associada com o risco global de doença cardiovascular, como doença cardíaca coronariana e acidente vascular cerebral ${ }^{18}$. É sugerido também que o alto consumo de laticínios está associado a menor prevalência de obesidade e que o cálcio pode ser um dos componentes que contribuem para a associação ${ }^{19}$. Além disso, sugere-se que existe uma significativa associação inversa entre a ingestão de produtos lácteos, laticínios de baixo teor de gordura, queijo e o risco de diabetes mellitus tipo $2^{20}$.

O consumo de carnes com excesso de gordura é um hábito não avaliado em outras pesquisas realizadas com universitários. A ingestão elevada de gorduras saturadas e alimentos ricos em colesterol proveniente do consumo habitual de gordura de origem animal aumenta o risco de doença coronariana, isquemia e outras doenças cardiovasculares ${ }^{21,22}$, sendo também considerada fator de risco para o desenvolvimento de dislipidemias ${ }^{23}$. Tais fatos alertam a necessidade de investigar o consumo excessivo de gordura animal e conscientizar os universitários quanto as consequências deste hábito.

Em relação a ingestão de bebidas alcoólicas, foi possível encontrar quatro estudos ${ }^{5,8,9,17}$, cuja prevalência de consumo foi menor do que a verificada na presente pesquisa. A relação entre esta ingestão e a doença cerebrovascular tem sido fortemente estabelecida, tanto na forma oclusiva quanto na hemorrágica ${ }^{9}$, além de que o consumo por períodos prolongados de tempo pode aumentar a pressão arterial e a mortalidade cardiovascular ${ }^{24}$, contribuindo também possivelmente para uma maior adiposidade corporal ${ }^{25,26}$. Assim, é necessário a criação de programas educativos voltados para a redução do consumo de bebidas alcoólicas devido à sua prevalência alarmante na população estudada.

Apesar de existirem diversos fatores de motivação para a prática de exercícios físicos em universitários, como a prevenção de doenças e controle do peso corporal ${ }^{27}$, percebeu-se elevada prevalência de comportamentos sedentários na população universitária avaliada, tanto no que se refere a ausência/carência de exercícios físicos quanto ao gasto excessivo de tempo com eletrônicos. Sabe-se que, o número de horas diárias que o indivíduo despende assistindo televisão, por exemplo, está associado ao consumo de alimentos calóricos, refrigerante e baixo consumo de frutas e hortaliças, além de pouco gasto de energia ${ }^{28}$.

De maneira geral, no presente estudo encontrou-se frequências mais altas de condutas de risco nos homens, o que é preocupante, pois segundo o Ministério da Saúde ${ }^{29}$, estudos comparativos entre homens e mulheres comprovaram que os homens são mais vulneráveis às doenças, principalmente as enfermidades graves e crônicas, e morrem mais precocemente que as mulheres.

Ainda quanto aos gêneros, o hábito de fumar encontrado com maior prevalência nos homens corrobora com diversos estudos realizados com universitários 17,30,31, além do baixo consumo de frutas ${ }^{17}$. Semelhantemente, adultos brasileiros do sexo masculino apresentam frequentemente o hábito de fumar, excesso de peso, consumo regular de refrigerantes com açúcar e hábito de consumo de carnes com gordura visível $^{32}$.

A ingestão excessiva de doces e o baixo consumo de feijão realizado predominantemente pelas mulheres apresentam em concordância com outra pesquisa ${ }^{7}$, reforçando assim a necessidade de intervenções específicas para cada sexo, de acordo com os fatores de risco mais prevalentes em cada um deles.

Outro aspecto investigado no presente estudo foi a existência ou não de diferença entre alunos no início e final dos cursos. Em uma pesquisa realizada com estudantes de medicina ${ }^{33}$, percebeu-se que os alunos que estavam no início do curso, quando comparados àqueles do final, apresentaram significativamente menos tempo gastos por dia com atividades físicas moderadas e vigorosas. Estes achados vão ao encontro da presente investigação. Diferentemente, esta mesma pesquisa relata que o consumo de alimentos hipercalóricos é semelhante nos dois grupos, mantendo-se 
em níveis pouco saudáveis ${ }^{33}$, enquanto que no presente estudo foi encontrada uma predominância de ingestão excessiva de alimentos doces e bebidas açucaradas nos alunos que estão no início da vida universitária.

O excesso de peso significativamente maior nos alunos que estavam no final da faculdade, encontra-se em concordância com um estudo realizado a partir de dados do VIGITEL ${ }^{32}$, no qual menciona que comparativamente ao nível de mais baixa instrução, no nível mais alto a probabilidade de excesso de peso aumenta. Uma pesquisa feita com alunos de medicina ${ }^{34}$ observou que a prevalência de sobrepeso aumenta à medida que os universitários vão se aproximando do final do curso. Esse crescimento é um dos principais fatores de risco para doenças crônicas, uma vez que o risco delas aumenta diretamente com o grau de excesso de peso e acúmulo de gordura na região abdominal ${ }^{35}$.

Em relação às áreas de conhecimento, diversos estudos apresentam apenas o estilo de vida de universitários da área

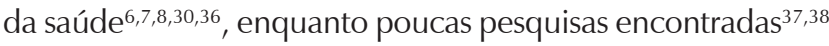
avaliam alunos de outras áreas de conhecimento. O hábito de fumar predominante entre estudantes das Ciências Humanas, Sociais, Aplicadas, Linguística, Letras e Artes, possivelmente é justificado pelo fato de apresentar-se como critério para inserção e aceitação de indivíduos em determinados grupos sociais 6 .

A prevalência encontrada do gasto excessivo de tempo com eletrônicos nas áreas da Engenharia, Ciências Exatas e da Terra, pode ser possivelmente explicada pela utilização frequente de computadores para cálculos, desenvolvimento e/ou utilização de softwares, entre outros. O computador, por exemplo, tem ampliado sua participação no meio acadêmico, pela imposição do mundo globalizado ou por iniciativas explícitas governamentais (através de programas de inclusão digital), tornando-se aos poucos uma ferramenta no aprendizado ${ }^{39}$. Desta forma, a criação de ferramentas virtuais de orientações para adoção de estilo de vida saudável seria de grande utilidade para melhoria da qualidade de vida desta população específica.

É importante salientar que também houve diferença significativa entre as áreas de conhecimento quanto ao baixo consumo de feijão e leite, além da ingestão excessiva de bebidas açucaradas. Porém, não encontrou-se dados na literatura a respeito dos fatores de risco em questão em estudantes de diferentes áreas de conhecimento, para comparar com os resultados observados no presente estudo.

Apesar do baixo consumo de leite ser menor entre estudantes das Ciências Biológicas, Agrárias e da Saúde, em relação às demais áreas, convém destacar que sua prevalência foi observada em mais da metade $(62,1 \%)$ dos estudantes no presente estudo, o que supera a prevalência encontrada em uma pesquisa feita especificamente com estudantes da área da saúde $(20,9 \%)^{7}$.

Uma vez que os universitários, em especial aqueles das Ciências da Saúde, serão formadores de opinião no exercício de suas profissões e considerados agentes multiplicadores de estilo de vida saudável, questiona-se os efeitos das condutas de saúde dos estudantes em sua prática futura.

É perceptível que os fatores de risco realmente tendem a se agruparem na população universitária. Uma pesquisa realizada também com universitários demonstrou que $86 \%$ dos mesmos apresentaram três ou mais fatores de risco ${ }^{9}$. Desta forma, a prevalência de simultaneidade dos comportamentos de risco encontrada no presente estudo $(95,4 \%)$ foi maior do que aquela verificada na literatura científica. Possivelmente isto se associa ao estilo de vida dos estudantes, visto que ao ingressarem na faculdade estão sujeitos a mudanças sociológicas e culturais, com consequências na adoção de hábitos ${ }^{5}$. Estes achados servem para confirmar a importância do monitoramento de tais comportamentos.

Não foi possível comparar com outras pesquisas a prevalência de fatores de risco agregados entre acadêmicos de diferentes gêneros, áreas de conhecimento e período do curso, uma vez que não foram encontrados estudos que abordassem tal questão. No entanto, destaca-se a importância de intervenções que visem reduzir a simultaneidade dos comportamentos de risco principalmente em estudantes homens e da área de Ciências Humanas, Sociais, Aplicadas, Linguística, Letras e Artes.

Dentre as limitações do estudo, convém mencionar que não foi realizado uma avaliação quantitativa dos alimentos abordados na pesquisa, bem como não se considerou o tipo, frequência e quantidade de ingestão de bebidas alcoólicas e do hábito de fumar. Em adição, o estudo não avaliou a relação causa e efeito, uma vez que não teve o caráter longitudinal.

Quanto ao estado nutricional, é importante destacar que, apesar da pouca utilização no Brasil de peso e altura referidos, possivelmente devido à deficiência de estudos nacionais e de base populacional analisando a validade destas informações, em outros países, o estudo da validade do peso e da altura referidos revelou alta correlação entre os valores mensurados e aqueles relatados ${ }^{40,41}$.

Existem dificuldades em comparar de forma precisa as prevalências dos fatores de risco encontradas no presente trabalho com os demais, pelo fato de existirem diferenças nas metodologias usadas, tanto na quantidade de fatores de risco selecionados quanto na definição dos mesmos. É necessária uma padronização dos instrumentos de coleta de dados em estudos realizados com acadêmicos, para que seja possível confrontar precisamente dados e acompanhar o estilo de vida desta população. 
Portanto, a população universitária apresentou elevada prevalência de fatores de risco para DCNT, requerendo assim a idealização, implantação e implementação de programas educativos voltados para a promoção da saúde e prevenção de doenças, a partir da adoção de um estilo de vida saudável que minimize a exposição dos universitários aos fatores de risco. Ademais, o presente estudo apresenta importância relevante por possibilitar a realização de intervenções específicas voltadas a cada gênero, área de conhecimento e período do curso.

\section{REFERÊNCIAS}

1. Ministério da Saude (BR). Secretaria de Vigilância em Saúde. Secretaria de Gestão Estratégica e Participativa. Vigitel Brasil 2011: vigilância de fatores de risco e proteção para doenças crônicas por inquérito telefônico. Brasília: Ministério da Saúde; 2012.

2. Schmidt MI, Duncan BB, Silva GA, Menezes AM, Monteiro CA Barreto SM, Chor D, Menezes PR. Doenças crônicas não transmissíveis no Brasil: carga e desafios atuais. Lancet. 2011;377:1949-61. http:// dx.doi.org/10.1016/S0140-6736(11)60135-9

3. Ministério da Saúde (BR). Secretaria de Vigilância em Saúde. Departamento de Análise de Situação de Saúde. Plano de ações estratégicas para o enfrentamento das doenças crônicas não transmissíveis (DCNT) no Brasil 2011-2022. Brasília: Ministério da Saúde; 2011

4. Ministério da Saúde. Secretaria de Atenção à Saúde. Departamento de Atenção Básica. Obesidade. Brasília: Ministério da Saúde; 2006.

5. Ledo-Varela MT, Román DAL, González-Sagrado M, Jauregui, OI, Vicente RC, Fuente RA. Características nutricionales y estilo de vida en universitarios. Nutr Hosp. 2011;26:814-18.

6. Cardoso DAP, Santos MLSC, Berardinelli LMM. A relação estilo de vida e tabagismo entre acadêmicos de enfermagem. Rev Eletr Enf [Internet]. 2009 [cited 2013 Mar 29];11(2):368-74. Available from: http://www.fen.ufg.br/revista/v11/n2/v11n2a18.htm

7. Paixão LA, Dias RMR, Prado WL. Estilo de vida e estado nutricional de universitários ingressantes em cursos da área de saúde do Recife/ PE. Rev Bras Ativ Fís Saúde. 2010;15:145-50.

8. Alves, EF. Estilo de vida de estudantes de graduação em enfermagem de uma instituição do sul do Brasil. Rev CPAQV. 2011;3(1):1-14.

9. Veras VS, Monteiro LZ, Landim CAP, Xavier ATF, Pinheiro MHNP, Montenegro RM. Levantamento dos fatores de risco para doenças crônicas em universitários. RBPS. 2007;20:168-72. http://dx.doi. org/10.5020/18061230.2007.p168

10. Silva KS, Lopes AS, Vasques DG, Costa FF, Silva RCR . Simultaneidade dos fatores de risco para doenças crônicas não transmissíveis em adolescentes: prevalênca e fatores associados. Rev Paul Pediatr. 2012;30:338-45. http://dx.doi.org/10.1590/S010305822012000300006

11. World Health Organization. Obesity: prevening and managing the global epidemic. Geneva: WHO; 1990.

12. Ministério da Saúde (BR). Protocolos do Sistema de Vigilância Alimentar e Nutricional - SISVAN na assistência à saúde. Brasília: Ministério da Saúde; 2008.
13. Ministério da Saude (BR). Secretaria de Vigilância em Saúde. Vigitel Brasil 2013: vigilância de fatores de risco e proteção para doenças crônicas por inquérito telefônico. Brasília: Ministério da Saúde; 2014.

14. Instituto Brasileiro de Geografia e Estatística. Pesquisa Nacional de Saúde Escolar, 2012. Rio de Janeiro: IBGE; 2013.

15. Pate RR, Pratt M, Blair SN, Haskell WL, Macera CA, Bouchard C, Buchner D, Ettinger W, Heath GW, King AC, Kriska A, Leon AS, Marcus BH, Morris J, Paffenbarger Jr RS, Patrick K, Pollock ML, Rippe JM, Sallis J, Wilmore JH. Physical activity and public health. A recommendation from the Centers for Disease Control and Prevention and the American College of Sports Medicine. JAMA. 1995;273:402-7. http://dx.doi.org/10.1001/ jama.1995.03520290054029

16. Ministério da Saúde (BR). Guia alimentar [Internet]: como ter uma alimentação saudável. 2006 [cited 2014 Out 21]. Available from: http://bvsms.saude.gov.br/bvs/publicacoes/guia_alimentar_ alimentacao_saudavel.pdf

17. Sousa TF, José HPM, Barbosa AR. Condutas negativas à saúde em estudantes universitários brasileiros. Ciênc Saúde Colet. 2013;18: 3563-75. http://dx.doi.org/10.1590/S1413-81232013001200013

18. Soedamah-Muthu SS, Ding EL, Al-Delaimy WK, Hu FB, Engberink MF, Willett WC, Geleijnse JM. Milk and dairy consumption and incidence of cardiovascular diseases and all-cause mortality: doseresponse meta-analysis of prospective cohort studies. Am J Clin Nutr. 2011;93:158-71. http://dx.doi.org/10.3945/ajcn.2010.29866

19. Lee HJ, Cho JI, Lee HSH, Kim Cl, Cho E. Intakes of Dairy Products and Calcium and Obesity in Korean Adults: Korean National Health and Nutrition Examination Surveys (KNHANES) 2007-2009. PLoS One. 2014 Jun 10;9(6):e99085. http://dx.doi.org/10.1371/journal. pone.0099085

20. Aune D, Norat T, Romundstad P, Vatten LJ. Dairy products and the risk of type 2 diabetes: a systematic review and dose-response metaanalysis of cohort studies. Am J Clin Nutr. 2013;98:1066-83. http:// dx.doi.org/10.3945/ajen.113.059030

21. Kris-Etherton P, Daniels SR, Eckel RH, Engler M, Howard BV, Krauss RM, Lichtenstein AH, Sacks F, St Jeor S, Stampfer M, Grundy SM, Appel LJ, Byers T, Campos H, Cooney G, Denke MA, Kennedy E, Marckmann P, Pearson TA, Riccardi G, Rudel LL, Rudrum M, Stein DT, Tracy RP, Ursin V, Vogel RA, Zock PL, Bazzarre TL, Clark J. AHA scientific statement: summary of the Scientific Conference on Dietary Fatty Acids and Cardiovascular Health. J Nutr. 2001;131:1322-26.

22. Mann JI. Diet and risk of coronary heart disease and type 2 diabetes. Lancet. 2002;360:783-9. http://dx.doi.org/10.1016/S0140-6736 (02)09901-4

23. Muniz LC, Schneider BC, Silva ICM, Matijasevich A, Santos IS. Fatores de risco comportamentais acumulados para doenças cardiovasculares no sul do Brasil. Rev Saúde Publ. 2012;46:53442. http://dx.doi.org/10.1590/S0034-89102012005000021

24. Sociedade Brasileira de Cardiologia; Sociedade Brasileira de Hipertensão; Sociedade Brasileira de Nefrologia. VI Diretrizes Brasileiras de Hipertensão. Arq Bras Cardiol. 2010;95:1-51.

25. Silva ABJ, Oliveira AVK, Silva JD, Quintaes KD, Fonseca VAS, Nemer ASA. Relação entre consumo de bebidas alcoólicas por universitárias e adiposidade corporal. J Bras Psiquiatr. 2011;60:210-15. http:// dx.doi.org/10.1590/S0047-20852011000300010 
26. Guimarães NS, Nemer ASA, Fausto MA. Influência do consumo de álcool nas alterações antropométricas: uma revisão sistemática. Nutr Clín Diet Hosp. 2013;33:68-76.

27. Guedes DP, Legnani RFS, Legnani E. Motivos para a prática de exercício físico em universitários de acordo com o índice de massa corporal. Rev Bras Ativ Fis Saúde. 2012;17:270-4.

28. Instituto Brasileiro de Geografia Estatística. Pesquisa Nacional de Saúde do Escolar. Rio de Janeiro: Ministério do Planejamento, Orçamento e Gestão; 2009.

29. Ministério da Saúde (BR). Política Nacional de Atenção Integral à Saúde do Homem (Princípios e Diretrizes) [Internet]. 2008; [cited 2014 Dez 04]. Available from: http://dtr2001.saude.gov.br/sas/ PORTARIAS/Port2008/PT-09-CONS.pdf

30. Marin NS, Rodrigues ART, Kinoshita NGC, Nakamura AS, Bueno PCS, Kinoshita SK. Tabagismo: caracterização do grau de dependência entre estudantes de medicina. RBSP. 2012;36:408-17.

31. Colares V, Franca C, Gonzalez E. Condutas de saúde entre universitários: diferenças entre gêneros. Cad Saúde Pública. 2009; 25:521-28. http://dx.doi.org/10.1590/S0102-311X2009000300007

32. Moura EC, Silva SA, Malta DC, Morais Neto OL. Fatores de risco e proteção para doenças crônicas: vigilância por meio de inquérito telefônico, VIGITEL, Brasil, 2007. Cad Saúde Pública. 2011;27: 486-96. http://dx.doi.org/10.1590/S0102-311X2011000300009

33. Faria DLS. Estudo Comparativo dos Estilos de Vida dos Estudantes de Medicina da Universidade da Beira Interior no Início e no Final do Curso [dissertação]. Covilhã: Universidade da Beira Interior; 2012.

34. Lessa SS, Montenegro AC. Avaliação da prevalencia de sobrepeso, do perfil nutricional e do nivel de AF nos estudantes de medicina da Universidade de Ciencias da Saude de Alagoas - UNCISAL. Rev Soc Bras Clin Med. 2008;6:90-3.

35. World Health Organization. Diet, nutrition and prevention of chronic diseases. Report FAO/WHO Expert Consulation. Geneva: WHO; 2003. (WHO Technical Report Series; vol. 916).

36. Monteiro MRP, Andrade MLO, Zanirati VF, Silva RR. Hábito e consumo alimentar de estudantes do sexo feminino dos cursos de Nutrição e de Enfermagem de uma universidade pública brasileira. Rev APS. 2009;12:271-77.

37. Andrade APA, Bernardo ACC, Viegas CAA, Ferreira DBL, Gomes TC, Sales MR. Prevalência e características do tabagismo em jovens da Universidade de Brasília. J Bras Pneumol. 2006;32:23-8. http:// dx.doi.org/10.1590/S1806-37132006000100007

38. Ramis TR, Mielke GI, Habeyche EC, Oliz MM, Azevedo MR, Hallal PC. Tabagismo e consumo de álcool em estudantes universitários: prevalência e fatores associados. Rev Bras Epidemiol. 2012;15: 376-85. http://dx.doi.org/10.1590/S1415-790X2012000200015

39. Fernandes L, Werner CML. Sobre o uso de jogos digitais para o ensino de engenharia de software [Internet]. [cited 2015 Jan 11]. Available from: http://fees.inf.puc-rio.br/FEESArtigos/artigos/artigos FEES09/FEES_3.pdf

40. Schmidt MI, Duncan BB, Tavares M, Polanczyk CA, Pellanda L, Zimmer PM. Validity of self-reported weight - A study of urban Brazilian adults. Rev Saude Publica. 1993;27:271-6. http://dx.doi. org/10.1590/S0034-89101993000400007

41. Spencer EA, Appleby PN, Davey GK, Key TJ. Validity of self-reported height and weight in 4808 EPIC-Oxford participants. Public Health Nutr. 2002;5:561-5. http://dx.doi.org/10.1079/PHN2001322 